\title{
Polar optical phonon states and their dispersive spectra of a wurtzite nitride superlattice with complex bases: transfer-matrix method*
}

\author{
L. Zhang ${ }^{\dagger}$ \\ Department of Mechanism and Electronics, Guangzhou Panyu Polytechnic, 511483 Guangzhou, People's \\ Republic of China
}

Received September 3, 2010, in final form October 31, 2010

Based on the dielectric continuum model and transfer-matrix method, the completing polar optical phonon states in a wurtzite GaN-based superlattice (SLs) with arbitrary-layer complex bases are investigated. It is proved that $2^{n}$ types of phonon modes probably exist in a wurtzite nitride SL with $n$-layer complex bases. The analytical phonon states of these modes and their dispersive equations in the wurtzite $\mathrm{GaN} / \mathrm{Al}_{x} \mathrm{Ga}_{1-x} \mathrm{~N} \mathrm{SL}$ structures are obtained. Numerical calculations on a three-layer $\mathrm{GaN} / \mathrm{Al}_{0.15} \mathrm{Ga}_{0.85} \mathrm{~N} / \mathrm{AIN}$ complex bases SL are performed. Results reveal that there are interface optical $(\mathrm{IO})$ phonon modes of one type only and four types of quasi-confined (QC) phonon modes in three-layer $\mathrm{GaN} / \mathrm{Al}_{0.15} \mathrm{Ga}_{0.85} \mathrm{~N} / \mathrm{AIN}$ complex bases SLs. The dispersive spectra of phonon modes in complex bases SLs extend to be a series of frequency bands. The behaviors of QC modes reducing to IO modes are observed. The present theoretical scheme and numerical results are quite useful for analyzing the dispersive spectra of completing phonon modes and their polaronic effect in wurtzite GaN-based SLs with complex bases.

Key words: optical phonon modes, dispersive spectra, wurtzite superlattice, transfer-matrix method

PACS: $63.22 . \mathrm{Np}, 68.65 . \mathrm{Cd}, 71.55 . \mathrm{Eq}$

\section{Introduction}

Since the superlattices (SLs) notion was first brought forward nearly four decades ago [1], the electronics and opto-electronics properties of semiconductor SLs have attracted considerable attention due to their fancy miniband effect and a lot of excellent physical properties [1, 2]. Among them, semiconductor SLs based on group-III nitride GaN, AlN, InN, and their ternary compounds $\mathrm{Al}_{x} \mathrm{Ga}_{1-x} \mathrm{~N}, \mathrm{In}_{x} \mathrm{Ga}_{1-x} \mathrm{~N}$ have invoked special interest both in theoretical and experimental investigations [3 -7]. This is mainly ascribed to the following evident characteristics: the nitride materials with widely and adjustable direct-band gap, strong atomic bonding and high carrier mobility make them attractive materials as a basis for the creation of reliable high-temperature and high-frequency electronic equipment and short-wavelength optoelectronics devices [5- 7 ].

In order to further improve the performance of microelectronics devices based on SL structures, people brought forward the complex-bases SL, i.e., $n$ layers per period $(n>2)[8-10]$. In contrast to the binary (two-layer per period) SLs, the complex bases SLs provide more degrees of freedom for the adjustable structural parameters, the miniband and minigap widths due to the introduction of additional layers in each SL period. This is very important for several important applications, such as infrared photodetectors, effective-mass filtering, and tuning of the tunneling current $11-13]$. The previous research on the complex bases SL is mainly focused on the electronic structures and surface polariton properties in GaAs-based semiconductors [14-19]. But there are few investigations of the other physical properties, such as the crystal-lattice dynamical feature and optoelectronic characteristics in GaN-based SL with complex bases [20]. Indeed, crystal-lattice vibrations (phonon

\footnotetext{
*The author would like to acknowledge the valuable guidance and discussion of Prof. J. Shi of Peking University. This work was supported by the National Natural Science Foundation of China for Youth under Grant No. 60906042.

${ }^{\dagger}$ E-mail: zhangli-gz@263.net
} 
modes) have great effect on the electronic and impurity states, the excitonic recombination and carrier transport, especially in low-dimensional quantum systems of polar semiconductors [21, 22]. At room and higher temperatures, the electron-phonon interactions and scattering by optical phonons play a dominant role effecting various properties of polar semiconductor quantum heterostructures, including hot-electron relaxation rates, interband transition rates, room-temperature exciton lifetimes, and many other optical and transport properties [23, 24]. Moreover, since nitride material usually crystallizes in the anisotropic hexagonal wurtzite structure, the crystal dynamical properties of nitride SLs are more complex due to anisotropy of the crystal structure in contrast to those of cubic crystals 25 32]. Hence, it is necessary and important to investigate the polar optical phonon modes in wurtzite GaN-based SL with complex bases.

On the basis of the dielectric continuum approximation and Loudon's uniaxial crystal model 33], several authors have made their great contributions to the study of the polar optical phonons and their electron-phonon interactions in wurtzite nitride binary SLs [20, 34, 35]. For example, Gleize and his coworkers [34] investigated the anisotropy effects on polar phonon modes in wurtzite GaN/AlN SLs, and the quasi-confined (QC) phonon modes and interface-optical (IO) phonon modes were found in the SL structures. Medeiros, Anselmo and their cooperators studied the confinement of polar optical phonons in two-layer periodic GaN/AlN SLs [35] based on the dielectric continuum model (DCM), and the phonon dispersive spectra of the systems were displayed in their discussions. From the viewpoint of experimental investigations, Darakchieva et al. [36] studied the phonons in AlN/GaN SLs using a combination of infrared spectroscopic ellipsometry and Raman scattering spectroscopy. And the $\mathrm{E}_{1}(\mathrm{TO}), \mathrm{A}_{1}(\mathrm{LO})$ and $\mathrm{E}_{2}$ localized, and $\mathrm{E}_{1}(\mathrm{LO})$ and $\mathrm{A}_{1}$ (TO) delocalized superlattice modes were identified. Recently, Davydov et al. [37] studied the lattice dynamics and Raman spectra of strained hexagonal wurtzite GaN/AlN and GaN/AlGaN SLs, and thus the IO and the QC phonon modes were observed. Gleize and coauthors [38] probed the confined phonons in hexagonal GaN-AlN artificial SL structures based on non-resonant Raman scattering, and the QC optical phonon modes were observed. Gleize's group [39] also measured the angular dispersion of polar IO and QC phonons in a hexagonal nitride SL, and the experimental data were in good agreement with the results of a previous calculation based on a DCM. Dutta et al. [40] reported Raman scattering results in binary SLs of GaN/(In)AlN, which also proves the availability of DCM for the description of polar optical phonon modes in wurtzite nitride heterostructures. Therefore, the DCM and Loudon's uniaxial crystal model are reliable and will be adopted to the investigation of the optical phonon modes in wurtzite nitride SL with complex bases in the present paper.

The main accomplishments and significance of this work lie in the following three points. (i) Via solving the Laplace equation of phonon potentials in wurtzite SLs with $n$-layer complex bases, it is confirmed that $2^{n}$ types of polar phonon modes, i.e. the propagating $(\mathrm{PR})$ mode, the IO mode, the $\left(2^{n}-2\right)$ QC modes probably coexist in wurtzite complex-base SL structures. (ii) By using the transfer-matrix method [8, 9], the polar optical phonon states and corresponding dispersive equations for the $2^{n}$ types of polar phonon modes in wurtzite nitride complex-bases SLs are given within the framework of the DCM and the Loudon's uniaxial lattice model. (iii) As an example, a wurtzite $\mathrm{GaN} / \mathrm{Al}_{x} \mathrm{Ga}_{1-x} \mathrm{~N} / \mathrm{Al}_{y} \mathrm{Ga}_{1-y} \mathrm{~N}$ SL with three-layer complex bases is chosen to check the theoretical scheme described in the present paper. The numerical result reveals that there are five types of phonon modes in the wurtzite $\mathrm{GaN} / \mathrm{Al}_{x} \mathrm{Ga}_{1-x} \mathrm{~N} / \mathrm{Al}_{y} \mathrm{Ga}_{1-y} \mathrm{~N}$ three-layer SLs chosen here. The dispersive spectra behave as a series of frequency bands, which are narrower than those in wurtzite two-layer GaN-based SLs [20, 34, 35]. The present theoretical scheme and numerical results are important and useful for further experimental and theoretical investigations of the polar phonon modes behaviors and their effect on optoelectronic properties in wurtzite nitride SLs with complex bases. The rest sections of the paper are organized as follows: the phonon states and their dispersive equations of a wurtzite GaN-based SL with $n$-layer complex bases are deduced in section 2 based on the transfer-matrix method; the numerical results for the dispersion relation, the electrostatic potential functions of the phonon modes on a wurtzite $\mathrm{GaN} / \mathrm{Al}_{x} \mathrm{Ga}_{1-x} \mathrm{~N} / \mathrm{Al}_{y} \mathrm{Ga}_{1-y} \mathrm{~N}$ SL with threelayer complex bases are performed and discussed in section 3; Finally, we summarized the main results and discussed the significance of the theory described in the current work in the last section. 


\section{Theory}

Let us consider a wurtzite $\mathrm{GaN} / \mathrm{Al}_{x_{1}} \mathrm{Ga}_{1-x_{1}} \mathrm{~N} / \mathrm{Al}_{x_{2}} \mathrm{Ga}_{1-x_{2}} \mathrm{~N} / \ldots / \mathrm{Al}_{x_{n-1}} \mathrm{Ga}_{1-x_{n-1}} \mathrm{~N}$ quasi-2dimensional (Q2D) complex bases SL with $n$-layer per period (as shown in figure 1). The $z$ axis is taken along the [0001] direction (c-axis) of the wurtzite crystals, and the origin of coordinate is chosen at the left hand side of some a GaN layer. The widths of GaN layers and the $\mathrm{Al}_{x_{i}} \mathrm{Ga}_{1-x_{i}} \mathrm{~N}$ layers are $d_{i}(i=0,1,2,3, \ldots, n-1)$, respectively. Thus the period of the complex bases SL is $L=\sum_{i=0}^{n-1} d_{i}$. For convenience, we label the GaN layer with origin of coordinate and its right $\mathrm{Al}_{x_{i}} \mathrm{Ga}_{1-x_{i}} \mathrm{~N}(i=1,2, \ldots, n-1)$ layers as the unit cell 0 . The periodic unit cells of the SL on the right hand side of the unit cell 0 are labeled successively as unit cell $1,2,3, \ldots$, while those on the left hand side of unit cell 0 are labeled as unit cell $-1,-2,-3, \ldots$ Due to the anisotropy of wurtzite crystals, the polar phonon frequencies and the dielectric function becomes direction dependent. If one denotes the direction of $c$-axis as $z$ and its perpendicular direction as $t$, the dielectric tensor can be written as

$$
\epsilon_{i}(\omega)=\left(\begin{array}{lll}
\epsilon_{t, i}(\omega) & 0 & 0 \\
0 & \epsilon_{t, i}(\omega) & 0 \\
0 & 0 & \epsilon_{z, i}(\omega)
\end{array}\right)
$$

where

$$
\epsilon_{t, i}(\omega)=\epsilon_{t, i}^{\infty} \frac{\omega^{2}-\omega_{t, \mathrm{~L}, i}^{2}}{\omega^{2}-\omega_{t, \mathrm{~T}, i}^{2}}, \quad \epsilon_{z, i}(\omega)=\epsilon_{z, i}^{\infty} \frac{\omega^{2}-\omega_{z, \mathrm{~L}, i}^{2}}{\omega^{2}-\omega_{z, \mathrm{~T}, i}^{2}}, \quad(i=0,1,2, \ldots, n-1) .
$$

Here $\omega_{z, \mathrm{~L}}, \omega_{z, \mathrm{~T}}, \omega_{t, \mathrm{~L}}$ and $\omega_{t, \mathrm{~T}}$ are the zone center characteristic frequencies of $\mathrm{A}_{1}(\mathrm{LO}), \mathrm{A}_{1}(\mathrm{TO})$, $\mathrm{E}_{1}(\mathrm{LO})$, and $\mathrm{E}_{1}$ (TO) modes, respectively. The subscripts $i=0,1,2, \ldots, n-1$ denote the $i$ th-layer $\mathrm{Al}_{x_{i}} \mathrm{Ga}_{1-x_{i}} \mathrm{~N}$ materials in any unit cell $N$, respectively.

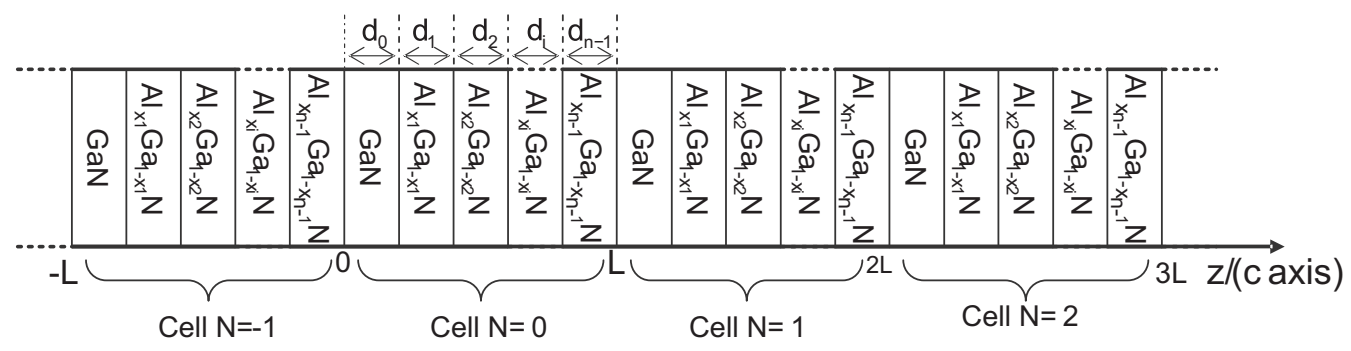

Figure 1. Schematic drawing of a GaN-based SL with $n$-layer complex bases. Here the $z$-axis is taken along the [0001] direction (c-axis).

In the case of free oscillations (the charge density $\rho_{0}(\mathbf{r})=0$ ) and using the Maxwell equations, the electric displacement vector $\mathbf{D}$ satisfies the following equation under the cylindrical coordinates, i.e.

$$
\nabla \cdot \mathbf{D}=-\varepsilon_{0}\left\{\epsilon_{t}(\omega)\left[\frac{1}{\rho} \frac{\partial}{\partial \rho}\left(\rho \frac{\partial}{\partial \rho}\right)+\frac{1}{\rho^{2}} \frac{\partial^{2}}{\partial \varphi^{2}}\right]+\epsilon_{z}(\omega) \frac{\partial^{2}}{\partial z^{2}}\right\} \Phi(\mathbf{r})=0 .
$$

It is obvious that equation (3) is a Laplace equation, and its solution $\Phi(\mathbf{r})$ is the electrostatic potential of polar optical phonon modes. In fact, via solving the equation (3) in the wurtzite SLs, one can get the complete polar optical phonon modes. In order to solve equation (3) conveniently, we define two functions as

$$
\begin{aligned}
\zeta_{i}(\omega) & =\operatorname{sign}\left[\epsilon_{z, i}(\omega) \epsilon_{t, i}(\omega)\right], \\
\gamma_{i}(\omega) & =\sqrt{\epsilon_{t, i}(\omega) / \epsilon_{z, i}(\omega),} \quad(i=0,1,2, \ldots, n-1), \\
\gamma_{i}(\omega) & =\left\{\begin{array}{cl}
\sqrt{\left|\epsilon_{t, i}(\omega) / \epsilon_{z, i}(\omega)\right|}, & \zeta_{i}(\omega) \geqslant 0, \\
\pm \mathrm{i} \sqrt{\left|\epsilon_{t, i}(\omega) / \epsilon_{z, i}(\omega)\right|}, & \zeta_{i}(\omega)<0 .
\end{array}\right.
\end{aligned}
$$


In terms of the theories of two-order differential equations, we know that, when $\zeta_{i}(\omega)<0$, the electrostatic potentials of phonon modes in the $i$ th-layer material correspond to the oscillating waves. On the contrary, they correspond to the decaying ones when $\zeta_{i}(\omega)>0[26$ 28]. Taking into account the oscillating or decaying waves in $n$-layers of some unit cell in the complex bases SL structures, it can be confirmed that $2^{n}$ types of polar phonon modes are likely to exist in wurtzite GaN-based SLs with $n$-layer complex bases. They are the IO phonon mode $\left(\zeta_{i}(\omega)>0\right)$, the PR phonon mode $\left(\zeta_{i}(\omega)<0\right)$ 25, 34], and the $\left(2^{n}-2\right)$ types of the QC phonon modes (at least one $\zeta_{i}(\omega)>0$, and one $\left.\zeta_{j}(\omega)<0, i \neq j\right)$ [34, 37, 39] in wurtzite SLs, respectively. These QC modes are distinguished and labeled as follows. In some unit cell of the complex bases SL, if the function $\zeta_{i}(\omega)$ takes negative values in $j=\mathrm{u}, \mathrm{v}, \mathrm{w}, \ldots(j<n)$ layers, then the QC mode is expressed by $\mathrm{QC}^{\mathrm{u}, \mathrm{v}, \mathrm{w}, \ldots}$. For example, in a three-layer complex bases SL, the QC modes are denoted as $\mathrm{QC}^{\mathrm{i}-\mathrm{ii}}$ if the functions $\zeta_{i}(\omega)(i=1,2)$ are negative, and $\zeta_{3}(\omega)>0$.

Next, let us discuss the phonon states and dispersive equations of all the phonon modes in wurtzite nitride SLs with $n$-layer complex bases in a uniform method. Considering the translational invariance in $\rho$-plane (the perpendicular plane to $z$ axis) of the SL quantum structures, the phonon electrostatic potentials in the $N$-th unit cell of the wurtzite GaN-based SLs with complex bases can be written as follows,

$$
\Phi_{N}(\mathbf{r})=\Phi_{N}(\rho, z)=\mathrm{e}^{\mathrm{i} \mathbf{k}_{t} \cdot \rho} \phi_{N}(z)
$$

and

$$
\begin{aligned}
\phi_{N}(z)= & \begin{cases}\phi_{N, 0}(z), & N L<z \leqslant N L+d_{0} \\
\cdots & \ldots \\
\phi_{N, i}(z), & N L+\sum_{i=0}^{i-1} d_{i}<z \leqslant N L+\sum_{i=0}^{i} d_{i} \\
\ldots & \ldots \\
\phi_{N, n-1}(z), & N L+\sum_{i=0}^{n-2} d_{i}<z \leqslant(N+1) L\end{cases} \\
= & \mathrm{e}^{\mathrm{i} q N L} \times\left\{\begin{array}{l}
A_{0} \exp \left[\gamma_{0} k_{t}(z-N L)\right]+B_{0} \exp \left[-\gamma_{0} k_{t}(z-N L)\right] \\
\ldots \\
A_{i} \exp \left[\gamma_{i} k_{t}\left(z-N L-\sum_{i=0}^{i-1} d_{i}\right)\right] \\
+B_{i} \exp \left[-\gamma_{i} k_{t}\left(z-N L-\sum_{i=0}^{i-1} d_{i}\right)\right] \\
\ldots \\
A_{n-1} \exp \left[\gamma_{n-1} k_{t}\left(z-N L-\sum_{i=0}^{n-2} d_{i}\right)\right] \\
+B_{n-1} \exp \left[-\gamma_{n-1} k_{t}\left(z-N L-\sum_{i=0}^{n-2} d_{i}\right)\right]
\end{array}\right.
\end{aligned}
$$

where $\mathbf{k}_{t}$ is a wave-vector of phonon modes in the $\rho$-plane. And $q$ is a wave vector that will arise in the dispersion relation for the collective excitations in SLs. In fact, the phase factor $\mathrm{e}^{\mathrm{i} q N L}$ is introduced to fulfill the Bloch's theorem: [41, 42]

$$
\begin{aligned}
\phi(z) & =\mathrm{e}^{\mathrm{i} q z} U_{q}(z), \\
U_{q}(z+N L) & =U_{q}(z), \quad(N=0, \pm 1, \pm 2, \ldots) .
\end{aligned}
$$

Via equation (4), it is seen that $\gamma_{i}(\omega)=\sqrt{\left|\epsilon_{t, i}(\omega) / \epsilon_{z, i}(\omega)\right|}$ as $\zeta_{i}(\omega)>0$. The potential $\phi_{N, i}(z)=$ $\mathcal{A}_{i} \exp \left[\gamma_{i} k_{t} z\right]+\mathcal{B}_{i} \exp \left[-\gamma_{i} k_{t} z\right]$ in the $i$-layer of unit cell $N$ behaves as a decaying wave based on equation (6) in this situation. On the contrary, $\gamma_{i}(\omega)= \pm \mathrm{i} \sqrt{\left|\epsilon_{t, i}(\omega) / \epsilon_{z, i}(\omega)\right|}$ as $\zeta_{i}(\omega)<0$. Under this situation, based on equation (6), it is found that the potential function $\phi_{N, i}(z)=$ $\mathcal{A}_{i} \exp \left[\mathrm{i}\left|\gamma_{i}\right| k_{t} z\right]+\mathcal{B}_{i} \exp \left[-\mathrm{i}\left|\gamma_{i}\right| k_{t} z\right]$ in the $i$-layer of unit cell $N$, which just behaves as an oscillating wave. This illustrates that, whether $\zeta_{i}(\omega)$ is positive or negative, the equation (6) gives the complete phonon electrostatic potentials of the phonon modes including the IO modes, the PR modes and the QC modes in wurtzite complex bases SLs.

The phonon potential functions and the normal components of electric displacement should be continuous at each heterostructure interface. These are referred to as continuity boundary conditions (BCs). Using these continuity BCs, one can obtain a series of BC Eqs. (8) in GaN-based SLs with complex bases, i.e.,

$$
\phi_{N, 0}(N L)=\phi_{N-1, n-1}(N L)
$$




$$
\begin{array}{rlll}
\left.\epsilon_{z, 0} \frac{\mathrm{d} \phi_{N, 0}(z)}{\mathrm{d} z}\right|_{z=N L} & = & \left.\epsilon_{z, n-1} \frac{\mathrm{d} \phi_{N-1, n-1}(z)}{\mathrm{d} z}\right|_{z=N L}, \\
\phi_{N, i}\left(N L+\sum_{i=0}^{i-1} d_{i}\right) & = & \phi_{N-1, i+1}\left(N L+\sum_{i=0}^{i-1} d_{i}\right), \\
\left.\epsilon_{z, i} \frac{\mathrm{d} \phi_{N, i}(z)}{\mathrm{d} z}\right|_{z=N L+\sum_{i=0}^{i-1} d_{i}} & = & \left.\epsilon_{z, i+1} \frac{\mathrm{d} \phi_{N-1, i+1}(z)}{\mathrm{d} z}\right|_{z=N L+\sum_{i=0}^{i-1} d_{i}}, \\
\phi_{N, n-2}\left(N L+\sum_{i=0}^{n-2} d_{i}\right) & = & \phi_{N, n-1}\left(N L+\sum_{i=0}^{n-2} d_{i}\right), \\
\left.\epsilon_{z, n-2} \frac{\mathrm{d} \phi_{N, n-2}(z)}{\mathrm{d} z}\right|_{z=N L+\sum_{i=0}^{n-2} d_{i}} & = & \left.\epsilon_{z, n-1} \frac{\mathrm{d} \phi_{N, n-1}(z)}{\mathrm{d} z}\right|_{z=N L+\sum_{i=0}^{n-2} d_{i}} .
\end{array}
$$

Substituting equation (6) into equations (8), and defining two $2 \times 2$ matrixes equations (9), (10) and a vector (11), the equations (8) can be expressed in concise forms. The matrixes and the vector are defined as follows:

$$
\begin{gathered}
\mathbf{M}_{i}=\left(\begin{array}{cc}
\exp \left(\gamma_{i} k_{t} d_{i}\right) & \exp \left(-\gamma_{i} k_{t} d_{i}\right) \\
\epsilon_{z, i} \exp \left(\gamma_{i} k_{t} d_{i}\right) & -\epsilon_{z, i} \exp \left(\gamma_{i} k_{t} d_{i}\right)
\end{array}\right) \\
\mathbf{N}_{i}=\left(\begin{array}{cc}
1 & 1 \\
\epsilon_{z, i} \gamma_{i} & -\epsilon_{z, i} \gamma_{i}
\end{array}\right)
\end{gathered}
$$

and

$$
\mathbf{C}_{i}=\left(\begin{array}{c}
A_{i} \\
B_{i}
\end{array}\right) \text {. }
$$

Using the above definitions (91) to (11), the equations (8) can be expressed compactly by the following matrix equations (12), i.e.,

$$
\begin{aligned}
& \exp (-\mathrm{i} q L) \mathbf{M}_{n-1} \mathbf{C}_{n-1}=\mathbf{N}_{0} \mathbf{C}_{0}, \\
& \text {... } \ldots . . . \\
& \mathbf{M}_{i} \mathbf{C}_{i}=\mathbf{N}_{i+1} \mathbf{C}_{i+1}, \quad(i=0,1, \ldots, n-2) \\
& \text {... } \ldots \ldots \\
& \mathbf{M}_{n-2} \mathbf{C}_{n-2}=\mathbf{N}_{n-1} \mathbf{C}_{n-1} .
\end{aligned}
$$

Via solving inverse matrix on equation (12), it is easy to get the relation:

$$
\exp (-\mathrm{i} q L) \mathbf{M}_{n-1} \mathbf{C}_{n-1}=\prod_{j=0}^{n-1} \mathbf{D}_{j} \mathbf{C}_{n-1},
$$

where

$$
\mathbf{D}_{j}=\mathbf{M}_{j}^{-1} \mathbf{N}_{j+1} .
$$

The condition of the linear and homogeneous equations (13) having nonzero solutions for $\mathbf{C}_{n-1}$ will result in $2 \times 2$ coefficient determinant equal to 0 , i.e.,

$$
\operatorname{Det}\left[\exp (-\mathrm{i} q L) \mathbf{M}_{n-1}-\prod_{j=0}^{n-1} \mathbf{D}_{j}\right]=0 .
$$

This equation (15) is just the dispersive equation of phonon modes in wurtzite nitride SLs with complex bases. Substituting the dielectric functions (2) and the structural parameters $d_{i}$ into the dispersive equation (15), the dispersive frequencies of phonon modes in the complex based nitride SLs can be worked out as a function of the wave-number $k_{t}$. It is worth to mention that, as the materials become isotropic (i.e. $\epsilon_{t}=\epsilon_{z}$ ), the dispersive equation (15) of anisotropic SL crystals will reduce to those of isotropic SLs [43]. In the situation, there are two types of phonon modes, i.e., the IO and the longitudinal-optical-like modes exist in the isotropic SL systems [21, 22, 43]. 


\section{Numerical results and discussion}

In section 2, we have derived the phonon states and their dispersive equations of all the polar optical phonon modes in wurtzite nitride SLs with $n$-layer complex bases. However, the corresponding analytical formulas are complicated. In order to get a clear picture for the dispersive behaviors of the types of polar optical phonon modes in wurtzite complex bases SL systems, an application of the present theories based on a nitride SL with $\mathrm{GaN} / \mathrm{Al}_{0.15} \mathrm{Ga}_{0.85} \mathrm{~N} / \mathrm{AlN}$ three-layer complex bases is performed, and a numerical calculation of dispersive relationship is carried out. The material parameters used in our calculations are listed in table 1 [26, 27, 34].

Table 1. Zone-center energies (in meV) of polar optical phonons, dielectric constants of wurtzite GaN, $\mathrm{Al}_{0.15} \mathrm{Ga}_{0.85} \mathrm{~N}$ and AlN materials [26, 27, 34].

\begin{tabular}{cccccc}
\hline & $\omega_{t, \mathrm{~T}}$ & $\omega_{t, \mathrm{~L}}$ & $\omega_{z, \mathrm{~T}}$ & $\omega_{z, \mathrm{~L}}$ & $\epsilon_{\infty}$ \\
\hline $\mathrm{GaN}$ & 69.25 & 91.83 & 65.91 & 90.97 & 5.35 \\
$\mathrm{AlN}$ & 83.13 & 113.02 & 75.72 & 110.30 & 4.77 \\
$\mathrm{Al}_{0.15} \mathrm{Ga}_{0.85} \mathrm{~N}$ & 71.332 & 90.009 & 67.382 & 93.87 & 5.263 \\
\hline
\end{tabular}

Table 2. Signs of the functions $\zeta_{i}(\omega)\left[i=1(\mathrm{GaN}), 2\left(\mathrm{Al}_{0.15} \mathrm{Ga}_{0.85} \mathrm{~N}\right)\right.$ and $\left.3(\mathrm{AlN})\right]$ and the frequency ranges of the phonon modes in wurtzite three-layer $\mathrm{GaN} / \mathrm{Al}_{0.15} \mathrm{Ga}_{0.85} \mathrm{~N} / \mathrm{AlN}$ complex bases SLs.

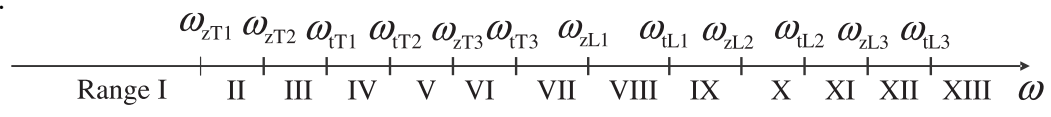

\begin{tabular}{llllllllllllllll}
\hline Ranges & I & II & III & IV & V & VI & VII & VIII & IX & X & XI & XII & XIII \\
\hline$\zeta_{1}(\omega)$ & + & - & - & + & + & + & + & - & + & + & + & + & + \\
$\zeta_{2}(\omega)$ & + & + & - & - & + & + & + & + & + & - & + & + & + \\
$\zeta_{3}(\omega)$ & + & + & + & + & + & - & + & + & + & + & + & - & + \\
Modes & IO & QC $^{\text {i }}$ & QC C $^{\text {i.ii }}$ & QC $^{\text {ii }}$ & IO & $\mathrm{QC}^{\mathrm{iii}}$ & IO & $\mathrm{QC}^{\mathrm{i}}$ & IO & $\mathrm{QC}^{\mathrm{ii}}$ & IO & $\mathrm{QC}^{\mathrm{iii}}$ & IO \\
\hline
\end{tabular}

Before analyzing the dispersive properties of phonon modes, let us first discuss the frequency ranges of the possible phonon modes in wurtzite SL systems with three-layer $\mathrm{GaN} / \mathrm{Al}_{0.15} \mathrm{Ga}_{0.85} \mathrm{~N} / \mathrm{AlN}$ complex bases. In terms of the material parameters given in table 1, the signs of the functions $\zeta_{i}(\omega)$ $\left[i=1(\mathrm{GaN}), 2\left(\mathrm{Al}_{0.15} \mathrm{Ga}_{0.85} \mathrm{~N}\right), 3(\mathrm{AlN})\right]$ and the frequency ranges of the phonon modes in wurtzite GaN-based SLs with three-layer complex bases are shown in table 2. From the table, it is observed that the characteristic frequencies of $\mathrm{GaN}, \mathrm{Al}_{0.15} \mathrm{Ga}_{0.85} \mathrm{~N}$ and $\mathrm{AlN}$ materials divide the frequency $\omega$ axis into thirteen subranges. For convenience, these subranges are labeled as range I, II, ..., XII, XIII from left to right. In each frequency subrange, the signs of the characteristic functions $\zeta_{i}(\omega)$ [equation (2)] are defined. Based on the properties of different types of phonon modes, namely behaving as decaying (vibrating) waves in the complex bases material layers of the SLs, the possible frequency ranges of these phonon modes in $\mathrm{GaN} / \mathrm{Al}_{0.15} \mathrm{Ga}_{0.85} \mathrm{~N} / \mathrm{AlN}$ complex bases SLs can be recognized. It is found that the IO modes of the system are likely to exist in the subranges I, V, IX, XI and XIII due to the positive values of $\zeta_{i}(\omega)(i=1,2,3)$ in these five subranges. The PR phonon modes do not appear in the $\mathrm{GaN} / \mathrm{Al}_{0.15} \mathrm{Ga}_{0.85} \mathrm{~N} / \mathrm{AlN}$ three-layer complex bases SLs because there are no frequency subranges satisfying simultaneously the conditions $\zeta_{i}(\omega)<0(i=1,2,3)$. It is found that four types of QC phonon modes, namely, the $\mathrm{QC}^{\mathrm{i}}, \mathrm{QC}^{\mathrm{i}, \mathrm{ii}}, \mathrm{QC}^{\mathrm{ii}}$ and $\mathrm{QC}^{\mathrm{iii}}$ phonon modes are likely to appear in the structure. Here the superscript "i, ii, iii" of $\mathrm{QC}^{\mathrm{i}, \mathrm{ii} \text {,iii }}$ modes represent $\mathrm{GaN}, \mathrm{Al}_{0.15} \mathrm{Ga}_{0.85} \mathrm{~N}$ and $\mathrm{AlN}$ in some unit cell of the nitride SLs, respectively. In fact, the $\mathrm{QC}^{\mathrm{i}}$ 
modes appear in ranges II and VIII, the $\mathrm{QC}^{\mathrm{i}, \mathrm{ii}}$ mode just exists in subrange of III, the $\mathrm{QC}^{\mathrm{ii}}$ modes also appear in two subranges of IV and X, and the frequency of $\mathrm{QC}^{\mathrm{iii}}$ modes falls into the subranges VI and XII. Taking into account the condition of solution [44], the dispersive equation (15) for IO phonon modes has no solution in the subranges I, VII and XIII. Thus, the IO phonon modes just appear in the three frequency subranges of V, IX and XI. And the subranges I, VII and XIII are termed as the forbidden frequency subranges. Apart from the forbidden frequency subranges I, VII and XIII of IO modes, the dispersive equations of other QC phonon modes have solutions in corresponding frequency ranges. Considering the forbidden subrange VII, the frequency subranges II, III,..., VI are termed as low-frequency range, while the subranges VIII, IX,..., XIII are referred to as high-frequency range.

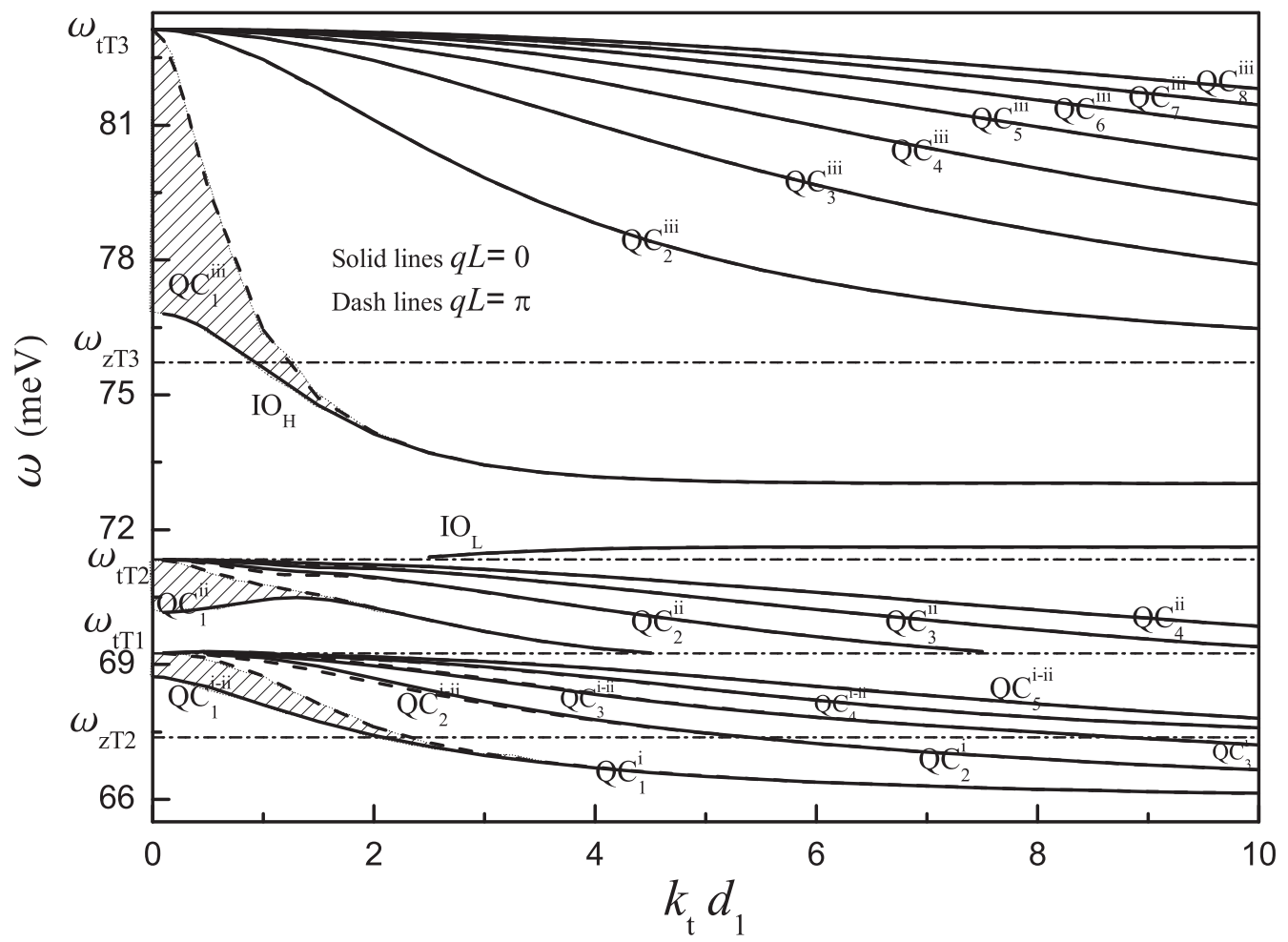

Figure 2. Dispersive spectra of the polar optical phonon modes in low-frequency as functions of the free wave-number $k_{t}$ of $\rho$-plane in a wurtzite three-layer $\mathrm{GaN} / \mathrm{Al}_{0.15} \mathrm{Ga}_{0.85} \mathrm{~N} / \mathrm{AlN}$ complex bases SL with $d_{1}=d_{2}=d_{3}=5 \mathrm{~nm}$. The solid lines and dashed lines correspond to the cases of $q L=0$ and $\pi$, respectively.

Figure 2 depicts the dispersive properties of the polar optical phonon modes in low-frequency range as a function of the free wave-number $k_{t}$ of $\rho$-plane in a three-layer $\mathrm{GaN} / \mathrm{Al}_{0.15} \mathrm{Ga}_{0.85} \mathrm{~N} / \mathrm{AlN}$ complex bases SL with $d_{1}=d_{2}=d_{3}=5 \mathrm{~nm}$. The solid lines and dashed lines correspond to the cases of phase $q L=0$ and $\pi$, respectively. The areas between a solid line and an adjacent dashed line are shaded. In fact, these shaded areas are the dispersive frequency bands of corresponding phonon modes in SLs. From the figure, it is clearly seen that four characteristic frequencies $\omega_{z, \mathrm{~T}, 2}$, $\omega_{t, \mathrm{~T}, 1}, \omega_{t, \mathrm{~T}, 2}$ and $\omega_{z, \mathrm{~T}, 3}$ (dashed and dot lines) divide the low-frequency range into five subranges. In terms of the order of increasing frequency, the $\mathrm{QC}^{\mathrm{i}}$, the $\mathrm{QC}^{\mathrm{i}-\mathrm{ii}}$, the $\mathrm{QC}^{\mathrm{ii}}$, the $\mathrm{IO}$ and the $\mathrm{QC}^{\mathrm{iii}}$ phonon modes appear successively in the five frequency subranges, respectively. These observations are completely consistent with the above analysis in table 2. Apart from the IO phonon modes, all the other phonon modes have infinity phonon branches in each frequency subrange. But only two branches of IO phonon modes exist in the low-frequency range, and they are labeled by $\mathrm{IO}_{\mathrm{L}}$ and $\mathrm{IO}_{\mathrm{H}}$ shown in the figure. For clarity, no more than six branches of QC phonon modes are plotted in each frequency subrange of QC modes here. These QC phonon branches are labeled by 
$\mathrm{QC}_{i}^{\mathrm{i} / \mathrm{i}-\mathrm{ii} / \mathrm{ii} / \mathrm{iii}},(i=1,2,3, \ldots)$, respectively. The subscript $i$ in symbol $\mathrm{QC}_{i}^{\mathrm{i} / \mathrm{i}-\mathrm{ii} / \mathrm{ii} / \mathrm{iii}}$ denotes the order of these QC phonon branches. In figure 1, all the other dispersive curves are monotonous and decreasing functions of $k_{t}$ except for the $\mathrm{IO}_{\mathrm{L}}$ modes and $\mathrm{QC}_{1}^{\mathrm{ii}}$ phonon branch. With the increase of $k_{t}$, the frequency bands (shaded areas) of phonon modes become narrower and narrower. And the solid lines and corresponding dashed lines converge to the same frequency values as $k_{t} d_{1}$ approaches 10. The profound physical reason for this feature lies in the obvious fact that, as the wave-number is large enough, the wave-length of phonon is quite short, and the complex bases SLs can be looked at as a series of independent three-layer $\mathrm{GaN} / \mathrm{Al}_{0.15} \mathrm{Ga}_{0.85} \mathrm{~N} / \mathrm{AlN} \mathrm{QWs}$. Thus, the phonon frequency bands of wurtzite SLs reduce to the phonon branches of wurtzite QWs. For example, the frequency of $\mathrm{IO}_{\mathrm{L}}$ modes of the SLs approaches $71.67 \mathrm{meV}$ as $k_{t} d_{1} \rightarrow 10$, which is just the same as the IO phonon limited frequency in GaN/AIN QWs 25, 27, 29]. Similarly, the $\mathrm{QC}^{\mathrm{i}-\mathrm{ii}}$ modes in the SLs will reduce to the analogous behaviors of $\mathrm{PR}$ phonon modes in $\mathrm{GaN} / \mathrm{Al}_{0.15} \mathrm{Ga}_{0.85} \mathrm{~N} \mathrm{QWs}[26]$. In a certain frequency subrange of some phonon modes, the higher is the order $i$ of $\mathrm{QC}_{i}^{\mathrm{i} / \mathrm{i}-\mathrm{ii} / \mathrm{ii} / \mathrm{iii}}$ $(i=1,2,3, \ldots)$, the weaker is the dispersion of the phonon modes. This is a general feature of phonon modes in low-dimensional quantum structures [26, 27, 34]. An interesting feature is that, as the frequencies of $\mathrm{QC}_{i}^{\mathrm{i}-\mathrm{ii}}$ phonon frequency bands are lower than the characteristic frequency $\omega_{z, \mathrm{~T}, 2}$, these $\mathrm{QC}_{i}^{\mathrm{i}-\mathrm{ii}}$ phonon bands degenerate into the corresponding $\mathrm{QC}_{i}^{\mathrm{i}}$ phonon frequency bands. Also, the $\mathrm{QC}_{1}^{\mathrm{iii}}$ degenerating to $\mathrm{IO}_{\mathrm{H}}$ is clearly observed. But the QC ${ }^{\mathrm{ii}}$ phonon modes are cut off at the frequency $\omega_{t, \mathrm{~T}, 1}$, which means that the number of the phonon branches decreases with the increase of $k_{t}$. These are the typical features of phonon modes in anisotropic wurtzite confined quantum systems [25 27, 34]. The extension of frequency bands (shaded areas) for the QC phonon modes is more distinct relative to those for the IO phonon modes in the low-frequency range.

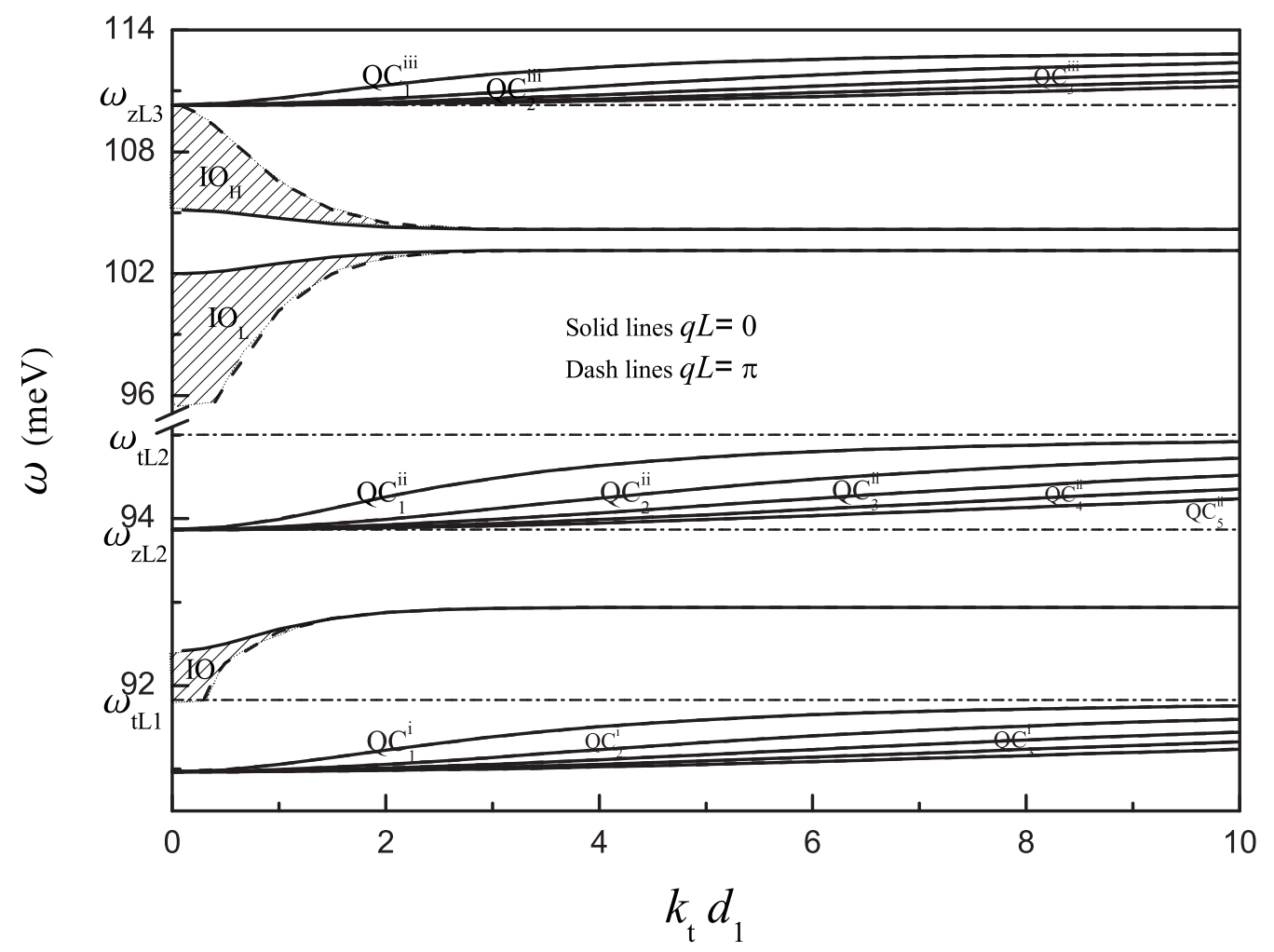

Figure 3. Dispersion frequencies $\omega$ of the polar optical phonon modes in high-frequency range as functions of $k_{t}$ in three-layer $\mathrm{GaN} / \mathrm{Al}_{0.15} \mathrm{Ga}_{0.85} \mathrm{~N}$ complex bases SL structures. The meanings of all curves and symbols in the figure are the same as those in figure 2.

The dispersive frequencies $\omega$ of the polar optical phonon modes in high-frequency range as functions of $k_{t}$ in a three-layer $\mathrm{GaN} / \mathrm{Al}_{0.15} \mathrm{Ga}_{0.85} \mathrm{~N} / \mathrm{AlN}$ complex bases SL are plotted in figure 3 . 
The meanings of all curves and symbols in the figures are the same as those in figure 2. Same as in figure 2, four characteristic frequencies $\omega_{t, \mathrm{~L}, 1}, \omega_{z, \mathrm{~L}, 2}, \omega_{t, \mathrm{~L}, 2}$ and $\omega_{z, \mathrm{~L}, 3}$ (dashed and dotted lines) divide the high-frequency range into five subranges. The $\mathrm{QC}^{\mathrm{i}}$ modes and $\mathrm{QC}^{\mathrm{i}}$ modes cover the lowest- and the highest- frequency subranges of the high-frequency range, respectively. The $\mathrm{QC}^{\mathrm{ii}}$ phonon modes occupy the middle subrange of $\left[\omega_{z, \mathrm{~L}, 2}, \omega_{t, \mathrm{~L}, 2}\right]$. These are the same as in figure 2 . But the IO phonon modes of high-frequency range appear in two subranges, which is quite different from those in figure 2. Except for the $\mathrm{IO}_{\mathrm{H}}$, the other dispersive curves are monotonous and increased functions of $k_{t}$. As $k_{t} d_{1} \rightarrow 10$, the IO phonon modes in the two subranges converge to three certain frequency values, which are the same value of $\mathrm{IO}$ phonon modes in $\mathrm{GaN} / \mathrm{Al}_{0.15} \mathrm{Ga}_{0.85} \mathrm{~N} / \mathrm{AlN}$ QWs [25, 27, 29]. The physics of this feature is analyzed above in figure 2. Comparing the frequency bands of phonon modes in the five subranges, it is found that the frequency band extensions of the IO modes in contrast to those of the QC modes in high-frequency range are quite obvious. This is just opposite to the case of the low-frequency range in figure 2 , in which the frequency band extensions of the QC modes are more distinct than those of IO phonon modes in low-frequency range. In fact, the frequency band extension of phonon modes is due to the periodic crystal structure of SLs, which is quite similar to the situation of energy band of periodic crystal structures [45]. As the wurtzite $\mathrm{GaN} / \mathrm{Al}_{x} \mathrm{Ga}_{1-x} \mathrm{~N} / \mathrm{AlN}$ SL reduces to the finite-layer GaN $/ \mathrm{Al}_{x} \mathrm{Ga}_{1-x} \mathrm{~N} / \mathrm{AlN}$ QW structures, the QC (IO) phonon bands in wurtzite SLs will naturally reduce to the dispersive curves of QC, PR, half-space (HS) and IO phonon modes in wurtzite QWs 25 29].

As stated in the Introduction, the complex bases SLs possess more adjustable structural parameters, and excellent miniband and minigap properties. These characteristics utilized in infrared photodetectors, effective-mass filtering, and tuning of the tunneling current 11 13. As the first step to investigate the polaronic effect on the optic and electronic properties in GaN-based SL with complex bases, the phonon modes and their dispersive spectra of the structures are necessary. As a new and important topic, the geometrical-parameter increases the polaronic effect of electronic and optoelectronic characteristics in GaN-based SLs with complex bases and will be studied and reported in the future. The IO and QC modes in binary GaN/AlN SLs were observed in experiments 37 39]. The experimental observations on phonon modes in GaN-based SLs with complex bases have been rarely reported by now due to the difficulty of crystal growth. With the great advance of semiconductor technology, the phonon modes are anticipated to be found in the GaN-base SLs with complex bases by the analogous techniques as those used in [37 39]. At last, we should point out that the present theoretical scheme and numerical results are based on non-retardation limit for simplicity. In fact, this treatment is widely adopted to be dealt with the crystal dynamics in GaN-based quantum structures [25-28, 33, 34]. The experimental results of angular dispersion of polar phonons and Raman scattering have been proven to be in good agreement with the calculations based on the non-retarded DCM [38, 39]. The recent calculation of polaronic binding energy in GaN nanowire also well agrees with relative experimental values [46]. This shows the reliability and meaningfulness of non-retardation treatment for the phonon modes in GaN quantum structures. Of course, a general case of retarded modes can be considered within the framework of transverse magnetic (TM) polarization [33].

\section{Conclusions}

In the present paper, we have analyzed and discussed the completing polar optical phonon modes in a wurtzite GaN-based SL with arbitrary-layer complex bases using the transfer-matrix method. Via solving the Laplace equations in wurtzite crystal, it is proved that $2^{n}$ types of polar phonon modes including the $\mathrm{PR}, \mathrm{IO}, \mathrm{QC}^{\mathrm{i} / \mathrm{ii} / \mathrm{iii}, \ldots}$ phonon modes are likely to exist in wurtzite nitride SLs with $n$-layer complex bases. The analytical phonon states of these phonon modes are obtained by means of the DCM and Loudon's uniaxial crystal model. Based on appropriate BCs, the concise dispersive equations of these phonon modes in wurtzite complex bases SLs are derived. An application of the present theories to a wurtzite SL with three layer $\mathrm{GaN} / \mathrm{Al}_{0.15} \mathrm{Ga}_{0.85} \mathrm{~N} / \mathrm{AlN}$ complex bases is performed. Numerical calculations on dispersive properties are carried out. Our results reveal that there are five types of phonon modes, i.e., one type of IO mode and four types of 
QC modes coexisting in the three-layer $\mathrm{GaN} / \mathrm{Al}_{0.15} \mathrm{Ga}_{0.85} \mathrm{~N} / \mathrm{AlN}$ complex bases SLs. The dispersive spectra of these phonon modes in SLs extend to be a series of frequency bands. It is also pointed out that, if the SL structures degenerate into corresponding finite wurtzite QWs, the QC and IO phonon bands in wurtzite SLs will naturally reduce to the PR, IO, HS and QC phonon modes in wurtzite QWs 25 29]. The present theoretical and numerical results are important for further analysis and discussion of the dispersive spectra of phonon modes and their polaronic effect on the optical and electronic properties in wurtzite $\mathrm{GaN} / \mathrm{Al}_{x} \mathrm{Ga}_{1-x} \mathrm{~N}$ SLs. We hope that the present work will stimulate further theoretical and experimental investigations of phonon properties, as well as their effect on the physical properties in wurtzite GaN-based SL systems.

\section{References}

1. Esaki L., Tsu R., IBM J. Res. Dev., 1970, 14, 61; doi:10.1147/rd.141.0061

2. Xia J.B., Zhu B.F., Semiconductor Superlattic Physics. Shanghai Science \& Technology Press, Shanghai, 1995.

3. Darakchieva V. et al., Phys. Stat. Sol. C, 2003, 0, 2614; doi:10.1002/pssc.200303408.

4. Avila-Ortega M.F., Pereyra P., Superlatt. Microstruct., 2008, 43, 645; doi:10.1016/j.spmi.2007.06.023

5. Gil B., Group III Nitride Semiconductor Compounds. Clarendon Press, Oxford, 1998.

6. Nakamura S., Chichibu S.F., Introduction to Nitride Semiconductor Blue Lasers and Light Emitting Diodes. Taylor \& Francis, London, 2000.

7. Yu L.S., Semiconductor Heterostructures Physics, 2nd ed., Science Press, Beijing, 2006.

8. Boudouti E.H.E., Djafari-Rouhani B., Akjouj A., Dobrzynski L., Phys. Rev. B, 1996, 54, 14728; doi:10.1103/PhysRevB.54.14728.

9. Djafari-Rouhania B., Dobrzynski L., Solid State Commun., 1987, 62, 609; doi:10.1016/0038-1098(87)90200-6

10. Esaki L., Chang L.L., Mendez E.E., Jpn. J. Appl. Phys., 1981, 20, L529; doi:10.1143/JJAP.20.L529

11. Yuh P., Wang K.L., Phys. Rev. B, 1988, 38, 13307; doi:10.1103/PhysRevB.38.13307

12. Vasilopoulos P., Peeters F.M., Aitelhabti D., Phys. Rev. B, 1990, 41, 10021; doi:10.1103/PhysRevB.41.10021

13. Choi K.K. et al., Phys. Rev. Lett., 1987, 59, 2459; doi:10.1103/PhysRevLett.59.2459.

14. Shi J.J., Pan S.H., Phys. Rev. B, 1993, 48, 8136; doi:10.1103/PhysRevB.48.8136

15. Boudouti E.H., et al., Phys. Rev. B, 1997, 56, 9603; doi:10.1103/PhysRevB.56.9603

16. Kucharczyk R.et al., Phys. Rev. B, 1998, 58, 5489; doi:10.1103/PhysRevB.58.4589.

17. Steslicka M. et al., Surf. Sci. Report, 2002, 47, 93; doi:10.1016/S0167-5729(02)00052-3

18. Cai M., Liu Y., Deng W., Phys. Rev. B, 1994, 49, 5429; doi:10.1103/PhysRevB.49.5429.

19. Mendialdua J. et al., Phys. Rev. B, 1994, 50, 14605; doi:10.1103/PhysRevB.50.14605

20. Medeiros S.K. et al., Solid State Commun., 2005, 135, 144; doi:10.1016/j.ssc.2005.02.027 Phys. Stat. Sol. C, 2005, 2, 2512; doi:10.1002/pssc.200461273

21. Shi J.J. et al., Phys. Rev. B, 1997, 55, 4670; doi:10.1103/PhysRevB.55.4670

22. Xie H.J., Chen C.Y., Ma B.K., Phys. Rev. B, 2000, 61, 4827; doi:10.1103/PhysRevB.61.4827.

23. Zhang X.B., Taliercio T., Kolliakos S., Lefebvre P., J. Phys.: Condens. Matter, 2001, 13, 7053; doi:10.1088/0953-8984/13/32/312

24. Balandin A.A., Pokatilov E.P., Nika D.L., J. Nanoelectr. Optoelectr., 2007, 2, 140; doi:10.1166/jno.2007.201

25. Shi J.J., Phys. Rev. B, 2003, 68, 165335; doi:10.1103/PhysRevB.68.165335.

26. Shi J.J., Chu X.L., Goldys E.M., Phys. Rev. B, 2004, 70, 115318; doi:10.1103/PhysRevB.70.115318.

27. Komirenko S.M., Kim K.W., Stroscio M.A., Dutta M., Phys. Rev. B, 1999, 59, 5013; doi:10.1103/PhysRevB.59.5013.

28. Li L., Liu D., Shi J.J., Eur. Phys. J. B, 2005, 44, 401; doi:10.1140/epjb/e2005-00139-x.

29. Zhang L., Shi J.J., Int. J. Mod. Phys. B, 2006, 20, 559; doi:10.1142/S0217979206033425

30. Zhang L., Shi J.J., Tansley T.L., Phys. Rev. B, 2005, 73, 245324; doi:10.1103/PhysRevB.72.199902.

31. Zhang L., Xie H.J., Shao P.M., Eur. Phys. J. B, 2010, 74, 397; doi:10.1140/epjb/e2010-00071-0

32. Zhang L., Condens. Matter Phys., 2010, 13, 13801.

33. Loudon R., Adv. Phys., 1964, 13, 423; doi:10.1080/00018736400101051.

34. Gleize J., Renucci M.A., Frandon J., Demangeot F., Phys. Rev. B, 1999, 60, 15985; doi:10.1103/PhysRevB.60.15985

35. Anselmo D.H.A.L. et al., Microelectron. J., 2005, 36, 407; doi:10.1016/j.mejo.2005.02.032. 
36. Darakchieva V. et al., Phys. Rev. B, 2005, 71, 115329; doi:10.1103/PhysRevB.71.115329

37. Davydov V.Y. et al., Phys. Stat. Sol. C, 2003, 0, 2035; doi:10.1002/pssc.200303311.

38. Gleize J. et al., Phys. Stat. Sol. A, 2001, 183, 157; doi:10.1002/1521-396X(200101)183:1<157::AID-PSSA157>3.0.CO;2-P

39. Gleize J. et al., Mater. Sci. Eng, B, 2001, 82, 27; doi:10.1016/S0921-5107(00)00683-8

40. Dutta M. et al., Physica E, 2001, 11, 277; doi:10.1016/S1386-9477(01)00217-X

41. Streight S.R., Mills D.L., Phys. Rev. B, 1987, 35, 6337; doi:10.1103/PhysRevB.35.6337

42. Tsuruoka T., Uehara Y., Ushioda S., Phys. Rev. B, 1994, 49, 4745; doi:10.1103/PhysRevB.49.4745.

43. Stroscio M.A., Dutta M., Phonons in nanostructures. Cambridge University Press, Cambridge, 2001.

44. The three dielectric functions $\epsilon_{i}(\omega)(i=1,2,3)$ (2) for $\mathrm{GaN}, \mathrm{Al}_{0.15} \mathrm{Ga}_{0.85} \mathrm{~N}$ and AlN materials take positive or negative values simultaneously in the frequency subranges of I, VII and XIII. For example, in the subrange I, all the functions $\epsilon_{j, i}(\omega)(j=z, t ; i=1,2,3)$ are positive. Under this situation, the dispersive equation (15) has no solution in the subranges I, VII and XIII. This means that the frequency subranges I, VII and XIII are not the frequency ranges of for IO phonon modes. Thus the IO modes just appear in the subranges of V, IX and XI.

45. Kittel C., Introduction to Solid State Physics. Chemical Industry Press, Beijing, 2005.

46. Zhang L., Shi J.J., Gao S., Semicond. Sci. Technol., 2008, 23, 045014; doi:10.1088/0268-1242/23/4/045014.

\title{
Стани полярних оптичних фононів та їхні дисперсійні спектри вюрцит нітридної супергратки зі складними базисами: метод трансфер-матриці
}

\author{
Л. Жанг \\ Політехніка Гуанчжоу Панью, Гуанчжоу, Народна Республіка Китай
}

На основі діелектричної неперервної моделі і методу трансфер-матриці, досліджено доповнені стани полярних оптичних фононів у вюрцит GaN-базованій супергратці (СГ) зі складними базисами. Доведено, що $2^{n}$ типи фононних мод існують у вюрцит нітридній СГ $3 n$-шаровими складними базисами. Отримано аналітичні фононні стани цих мод та їхні дисперсійні рівняння у структурах вюрциту $\mathrm{GaN} / \mathrm{Al}_{x} \mathrm{Ga}_{1-x} \mathrm{~N}$ СГ. Здійснено числові розрахунки на тришарових $\mathrm{GaN} / \mathrm{Al}_{0.15} \mathrm{Ga}_{0.85} \mathrm{~N} / \mathrm{AIN}$ складних базисах СГ. Результати показують, що є інтерфейсні оптичні (IO) фононні моди тільки одного типу і чотири типи квазіобмежених (КО) фононних мод у тришарових GaN/Al ${ }_{0.15} \mathrm{Ga}_{0.85} \mathrm{~N} / \mathrm{AIN}$ складних базисах суперграток. Дисперсійні спектри фононних мод в складних базисах суперграток розширюються до низки частотних зон. Спостерігається, що поведінка КО мод редукується до IO мод. Ця теоретична схема і числові результати $є$ досить корисними для аналізу дисперсійних спектрів доповнених фононних мод і їхніх поляронних впливів у вюрцит GaN-базованих супергратках із складними базисами.

Ключові слова: оптичні фононні моди, дисперсійні спектри, супергратка вюрциту, метод трансфер-матриці 
